\title{
Cryptocurrency Market Analysis: Realities and Prospects
}

\author{
Oleksandr Zaiets ${ }^{*}[0000-0002-4426-5241]$, Sergey Yeskov ${ }^{2}$ \\ ${ }^{1}$ Odesa State University of Internal Affairs, Odesa, Ukraine \\ ${ }^{2}$ Centre for European Reforms Studies, a.s.b.l. Grand Duchy of Luxembourg \\ *zaec_1985@meta.ua
}

\begin{abstract}
This article presents the analyze of the cryptocurrency market, the practice of state regulation of the cryptocurrency. The study of the cryptocurrency market and the experience of its regulation revealed certain similarities. Cryptogrivna, which can be put into circulation, means the formation of new institutional conditions for the development and functioning of the national financial market. At the same time, it is important to understand that cryptocurrency cannot completely replace traditional means of payment and payment methods, either in the medium or in the short term. In this regard, in order to ensure national economic security, the unification of the current tax and financial legislation with acts of international law, in terms of economic and organizational approaches to the circulation and emission of world and national cryptocurrencies, is of particular relevance. This will make it possible to create a number of new financial instruments that can be used to stimulate the growth of the national economy, which today is gradually emerging from the crisis zone, showing a trend towards further growth.
\end{abstract}

Keywords: market capitalizations, Bitcoin, cryptocurrency, blockchain, digitalization, digital economy, legal regulation.

\section{INTRODUCTION}

The world economy is in a permanent state of transformation: technological structures, material values, and regulation mechanisms are changing. This process can be accelerated by the emergence of new factors that change the fundamental foundations of the modern market economy.

Historically, money is centrally managed almost everywhere states through central state banks, starting from their issue and ending with the rules circulation, denomination and disposal. Undoubtedly there is a positive side to this, but there is also a negative one. At the same time, you need to understand that legal norms are secondary to social, which, in turn, appear in connection with certain phenomena, arising, in fact, in a legal vacuum. So it was with the advent of radio, cars, the Internet and many other achievements of scientific and technological progress. Same is happening now with new alternative money - cryptocurrencies - products of innovative blockchain technologies.

Currently, such a factor is global digitalization, which has influenced the development of almost all spheres of the economy. As a result, new markets, new forms of communication, new approaches to organizing and managing the production of goods and the provision of services have emerged. Active digitalization has led to the formation of a digital profile of a person, characterizing all aspects of his life, and also contributed to certain worldview changes - along with material or "tangible" values, digital ones have appeared: starting from rights to intellectual property (music, films, applications, etc.) and ending with characters in computer online games. These goods and services began to be "produced", bought and sold in the global digital marketplace, which since its inception had no geographic boundaries. A feature of this market is that the vast majority of goods and services are consumed and used in the digital space. This results in faster communication and "delivery" of digital products between sellers and buyers. At the same time, the servicing of purchase and sale transactions was (and is) carried out using fiat money and banking infrastructure. Understanding the essence and popularity of this phenomenon requires a more detailed consideration of the prerequisites for the emergence of cryptocurrency and the motives of economic entities (citizens, firms, banks and countries) who began to use it. 
Among the most popular cryptocurrencies in the world, the leaders of growth were Bitcoin, Ethereum, DASH, Monero and NEM - they own $91 \%$ of the total capitalization [1]. The price of the first cryptocurrency bitcoin - "has reached an absolute historical maximum and is already approaching the mark of 51000 dollars. for 1 BTC (exceeding the price of a troy ounce of gold)".

States face new regulatory challenges cryptocurrency transactions and other products of blockchain technologies, using positive sides with acceptable risks, embedding these blockchain innovations in current financial and legal system. Prohibition or permission, as well as complete ignoring is just a disclaimer.

\section{RESEARCH METHODOLOGY}

To solve the set tasks, the following methods were used: system and comparative analysis, synthesis, analysis of statistical data, deduction.

Publications, scientific publications, and textbooks were used as an information base for the study.

This study is a quantitative research to have a better understanding of crypto-currency speculative bitcoin price drivers. Firstly, some empirical analyses.

The basic goal of this study is to explore the knowledge of people about blockchain and the way this network works. This paper also covers some points of blockchain generations like bitcoin. In first section, the literature review has been discussed. A small knowledge about blockchain and its generations (bitcoin, ethereum) is mentioned. Then comparison of blockchain, bitcoin related to some aspect has been shown in form of table. Then result of comparison is discussed. At last, conclusion of whole study is defined.

\section{RESEARCH RESULTS}

Taking these prerequisites into account, the use cryptocurrencies and blockchain technologies have become one of the responses to the opportunities and challenges of the development of the world economy, incl. related to imperfection of state and market regulation: cryptocurrency is a product of the digital economy; when creating a cryptocurrency, algorithms based on objective mathematical laws are used; control over the circulation of cryptocurrencies is carried out by the system itself, which makes it more stable in relation to the actions of third parties; cryptocurrencies can reduce transaction costs; cryptocurrencies are an international product created in the interests of and to serve all participants in the settlement. Despite the technical limitations and difficulties associated with mining and the circulation of cryptocurrencies (low transaction processing speed, the risk [2] of system stability, etc.), individual states are already experimenting with digital money, as well as blockchain technology [3]. However, the significance of the prospects for using cryptocurrencies for citizens, government and business is different.
While a dynamic cryptocurrency market can revolutionize the way money is exchanged, its introduction to global platforms can be fraught with problems and potential errors. Since virtual currencies are not generally accepted as official means of payment for goods and services, developing standardized systems for their use is critical. For currencies to be stable, it is necessary to establish their legal status. Regulatory systems are evolving and different governments are taking a variety of approaches. The current regulatory measures are in their primary state and continue to evolve along with the booming cryptographic means of payment industry.

From the point of view of business, this is, first of all, a different format of interaction between economic agents with a reduction in the number of intermediaries represented by banks, state and quasi-state institutions. In practice, this means minimizing bureaucratic formalities for making settlements, increasing the speed of providing services, and reducing the cost of transactions. The irreversibility of transactions can be of particular value in comparison with the possibility of canceling any banking transaction or challenging and terminating transactions in court. The issuance of cryptocurrencies, digital signs (tokens) is also considered as a convenient way to attract private investments to finance the launch and / or financial support of current business projects.

Individuals approach cryptocurrencies and blockchain from various aspects: cheap instrument for large settlements; earning income through investments in the purchase of cryptocurrency and mining.

The growing interest of the population in cryptocurrency was largely due to the possibility of investing money and obtaining superprofits due to the growth of cryptocurrency quotes (primarily bitcoin). At the same time, many experts believe that investing in digital money is a "financial pyramid", and soon the financial bubble should burst or has already burst. The factors of the dynamics of the value of the cryptocurrency are poorly predictable, which is reflected in the significant jumps in the rate of the cryptocurrency in relation to fiat money. Despite the fact that the asset is not backed by real value, the potential profitability due to purchase and sale operations is very high, which makes investments in cryptocurrencies very risky and at the same time attractive for speculative capital. You can talk about the "overvalued" or "underestimated" of cryptocurrencies, but at the same time it is necessary to understand that these terms are applicable to a greater extent to fiat money. For the state, this issue is more complicated than for other subjects of the economy. On the one hand, there is great potential for the development of financial technologies. The use of cryptocurrencies and blockchain technology can simplify and speed up interbank and interstate settlements, the issuance and circulation of debt securities, the identification procedure, reduce the cost of transactions, ensure the security and convenience of transactions through the use 
of cryptography. The potential for using blockchain technology is also significant from the point of view of providing public services (for example, in transactions with confirmation of property rights).

The other side of the use of digital money [4] is related to the role of the state in the monetary system. Acting as the only issuer of legal banknotes, the state also controls the amount of money in the economy, its circulation and monetary inflation channels.

Cryptocurrency services (Bitcoin, Litecoin, Ethereum, Dash, Monero, etc.) have made a powerful step forward in a short time. Now users do not have to lose money on transactions through intermediaries, paying interest. You can use the crypto wallet on your computer or save money online on a specific service. However, it is better to keep money, third-party services often break, and everything is lost. It is logical to download the necessary software from the site (for example, from "Bitcoin") and install it on your computer based on the operating system [5]. It is better to choose full versions, rather than "light" options - it requires disk space, time and traffic, but this option is more reliable.

Cryptocurrency in Ukraine can be earned, bought or donated. It used to be easier to earn it, in 2017 the situation changed [6]. In Ukraine, "it is problematic to pay with cryptocurrency for goods or services, only units of online stores and businesses indicate on their sites that accept cryptocurrency as payment for goods or services" [7]. The business owner can use the payment in a new type of currency, as do many Western stores [8].

In some countries, employers are already paying their employees in cryptocurrency, usually to those who work online - in Ukraine, this practice is just beginning to spread. Wages in cryptocurrencies among Ukrainians today are mostly received by IT specialists working for foreign companies.

Sometimes cryptocurrency can be earned for free: it is a small amount of gift that attracts people around the world. All you have to do is enter a captcha or view an advertisement, and for this you will be charged, for example, Satoshi (one hundred million bitcoin). But this is not a normal way to earn money, because if the site is blocked, the cryptocurrency disappears. Gifts are possible from large services, for example, for a good post or article, a picture that is in demand among other users [9].

You can buy cryptocurrency in Ukraine on various exchanges, in exchangers. Exchangers are not always profitable, because they earn on the difference in value, the best options are with exchanges or buying directly from a seller with a good reputation [7]. It is important to remember the security of the transaction; many practitioners [6;3] recommend buying cryptocurrency from sellers in their city.
The process of creating a new payment instrument, which is cryptocurrencies, "uses the computer power of millions of participants, based on data from open ewallets" [10], which are combined into one peer-to-peer network in the absence of a central server. Thus, all work on accounting, storage of transaction history is distributed among all participants [11].

One of the most important aspects of the functioning of cryptocurrency is powerful modern cryptographic methods of protection that provide identification of owners and recording the fact of their change. Belief in the impossibility of breaking this protection seems to us somewhat idealistic without the proper constant IT support of specialists [12].

Given the current situation in Ukraine, ignoring the cryptocurrency market by the state is not a productive way to resolve the situation [13]. In our opinion, it is constructive to consider this phenomenon as an alternative to the existing system or as a supplement to it.

At the beginning of 2017, the most common cryptocurrencies in Ukraine were:

Bitcoin (BTC) is the first cryptocurrency created, confidently holding the palm of supremacy in the world and in the Ukrainian market. The price of the coin is approaching 50000 dollars. USA.

Ethereum (ETH) is a cryptocurrency that was issued only in 2015 on the basis of Ethereum technology, which belongs to the blockchain systems based on the use of distributed databases. Ethereum can be used in various fields, and is also a currency, an alternative to traditional instruments of the foreign exchange market. The price is about 1814 dollars. USA. Growing interest in this cryptocurrency has been facilitated by the ability to trade the ETH / USD pair, the world's largest social trading and investment network eToro, as well as the blockchain of the Enterprise Ethereum Alliance, which includes Microsoft, Intel and Accenture.

Analysts attribute the record-breaking growth of the cryptocurrency Dash to the release of the update Dash v12.1 - "Sentinel", designed to increase the speed of work, improve the levels of privacy and coordination in the network. The price of Dash today approached \$ 1814. USA.

Monero (XMR) is an open source cryptocurrency that is secure and anonymous. Stably included in the top five coins with a high market capitalization. Price - 245 dollars. USA.

Ripple (XRP) is an open source payment system, where the internal payment unit is the cryptocurrency of the same name. Ripple technology is based on the "principle of trust". The idea is to be able to exchange your currency for any other at the best rate in one click, adding the right gateway. Price - \$ 544. USA 
To understand the collective concept of "cryptocurrency", it should be borne in mind that all noncash (electronic) money (traditional fiat currencies) is also digital - cashless dollar, euro, hryvnia, webmoney, yandexmoney. The only difference is that in one case, the issue is centralized and far from always effectively managed by the National Bank, a municipality, an ordinary commercial bank, a financial company, and in the other - by the management of the issue of alternative money decentralized and not burdened with loan interest, immobilization tax, commissions and fees of the issuer.

\section{DISCUSSION OF RESULTS}

Many scientists have devoted their work to the study of the problems of creating a unified world payment system. Discussions are being held on ways to create an information society. Issues related to the further development of the digital economy as a digital ecosystem of the future society are also discussed. Research is being conducted on the impact of cryptocurrency technology on the financial side of the digital economy. The possibility of using blockchain technology as a unified digital platform for the digital economy of the future has been substantiated; the issue of expanding the scope of blockchain technology is being studied [1, p. 12].

Note that electronic payment instruments act as the newest variety of payment instruments, generated mainly by scientific and technological progress, rather than by the evolution of monetary and financial relations. At the same time, this term, like some other concepts (for example, "electronic settlements", "electronic money", etc.) do not have an unambiguous and clear definition in theory [9]. The materials are devoted to the analysis of the main properties of cryptocurrencies using the example of bitcoin (Bitcoin), which have a significant impact on the economy in the XXI century, as well as the problems of licensing cryptocurrencies in Ukraine and ways to solve this problem.

The competitiveness of modern banking structures operating in the market is directly dependent on the introduction of new banking technologies and products. Scientists give the characteristics of the bitcoin cryptocurrency, consider its advantages and disadvantages, as well as the specifics of bitcoin technology [14].

According to Santander-Bank, the use of a distributed ledger by banks will provide an opportunity to achieve significant savings on infrastructure (up to $\$ 20$ billion annually by 2022). Such registries will be cheaper to operate than those in use today and will prevent assets from being frozen for the hours or days that transfer transactions take today. The problems associated with the control over the turnover and emission of cryptocurrencies are considered in parallel with the concept of cryptocurrency and the general properties of these currencies.

As retailers around the world begin to formally respond to the virtual currency market, the scale of the currency's success ultimately depends on public acceptance. This path is not easy and will take a huge amount of effort to calm skeptical people. The physical value of a cryptocurrency lies in the number of its users. Without public trust, the virtual currency system as an alternative payment method will be unstable.

Gradually, thanks to the information background that defends its dignity, the cryptocurrency is gaining a presence in the world market. However, despite the recent surge in media coverage, cryptocurrencies are still widely unknown to the wider population of the planet. This trend is indicative of skepticism about Bitcoin's usefulness.

\section{CONCLUSIONS}

So, at present there are over a thousand different cryptocurrencies that require the development of a single standard for their regulation, adaptation of legislation in the tax area, as well as identification of cryptocurrencies to generate income.

In the process of regulating cryptocurrencies, states strive to create a favorable climate for the development of new technologies (blockchain), seeing the enormous potential for the use of technologies in various sectors of economic and social activity, as well as the potential for using cryptocurrencies for investment.

Based on the research conducted, many authors see directions for further development in the study of the possibilities of legal regulation, the emission of a national cryptocurrency, as well as its use to finance targeted programs, taking into account its advantages.

Cryptogrivna, which can be put into circulation, means the formation of new institutional conditions for the development and functioning of the national financial market. At the same time, it is important to understand that cryptocurrency cannot completely replace traditional means of payment and payment methods, either in the medium or in the short term.

In this regard, in order to ensure national economic security, the unification of the current tax and financial legislation with acts of international law, in terms of economic and organizational approaches to the circulation and emission of world and national cryptocurrencies, is of particular relevance. This will make it possible to create a number of new financial instruments that can be used to stimulate the growth of the national economy, which today is gradually emerging from the crisis zone, showing a trend towards further growth. 


\section{REFERENCES}

[1] Bauer, V.P. (2017), Problems in the way of creating a unified digital platform for the digital economy, RANS, Moscow, Russia, 39 p.

[2] Korystin, O.Ye. and Svyrydyuk, N.P. (2020), "Methodological principles of risk assessment in law enforcement activity", Nauka i pravooxorona, vol. 3, pp. 191-197. DOI: https: //doi.org/10.36486/np.2020.3(49).19

[3] Raihana Syahirah Abdullah, Faizal M.A. (2018), "Block Chain: Cryptographic Method in Fourth Industrial Revolution", International Journal of Computer Network and Information Security, vol. 10, No. 11, pp. 9-17, 2018. DOI: 10.5815/ijcnis.2018.11.02

[4] Tkachenko, Volodymyr Korystin, Oleksandr Voloshchuk, Taras Nekrasov, Viacheslav and Svyrydiuk, Natalia 2019 "Cryptocurrency Circulation in the Global and Domestic Financial Systems", Journal of Legal, Ethical and Regulatory Issues, Vol. 22, Special Issue 2, pp. 1-6, available at: https://www.abacademies.org/articles/ Cryptocurrency-circulation-in-the-global-anddomestic-financial-systems-1544-0044-22-SI-2363.pdf

[5] Ibrahim S.I. Abuhaiba1, Hanan M. Abuthraya, Huda B. Hubboub, Ruba A. Salamah (2012), "Image Encryption Using Chaotic Map and Block Chaining", International Journal of Computer Network and Information Security, No 7, pp. 19-26. DOI: $10.5815 /$ ijcnis.2012.07.03

[6] Korobeinikova, O.M. Korobeinikov, D.A. and Nazarbayev, O. (2017), "Innovative payment instruments in payment systems", Actual problems of humanitarian and socio-economic sciences, vol. 5, I. 11 (11), pp. 102-104.

[7] Akin Oyedele (2017), Banning of Bitcoin in China Retrieved From Businessinsider, Com. 13.

[8] Kevin Helms (2017), Ukraine's New Bills on BITCOIN Retrieved from, available at: www.Bitcoin.Com.

[9] Kupriyanovsky, V. P. "Economics of Digital Railroad Innovations. Experience of Great Britain", International Journal of Open Information Technologies, 2017, vol. 5, I. 3, pp. 79-99.

[10] Scheele, M. Initial coin offerings - asking the right regulatory questions, available at: https://talkingtech.cliffordchance.com/ content/micro-cctech/en/fintech/initial-coinofferings/_jcr_content/text/parsysthumb.pdf
[11] Savinsky, S.P. "Crypto-currencies and their regulatory and legal regulation in the PRC", Money and credit, 2017, № 7, pp. 65-67.

[12] Sidra Anwar, Sadia Anayat, Sheeza Butt, Saher Butt, Muhammad Saad (2020), “Generation Analysis of Blockchain Technology: Bitcoin and Ethereum", International Journal of Information Engineering and Electronic Business(IJIEEB), vol. 12, No.4, pp. 30-39. DOI: 10.5815/ijieeb.2020.04.04

[13] Korystin, O.Y. Svyrydiuk, N.P. Mihus, I.P. Likhovitskyy, Y.O. and Mitina, O.M. (2020), "Money laundering: macroeconomic assessment methods and current trend in Ukraine", Finansovokredytna diyal'nist': problemy teoriyi ta praktyky, vol. 1 (32), pp. 341-350. DOI: https://doi.org/10.18371/fcaptp.v1i32.20086 5

[14] Auqib Hamid Lone, Roohie Naaz Mir (2018), "Investigating and Analyzing Bitcoin Blockchain Protocol using Wireshark", International Journal of Computer Network and Information Security, vol. 10, No. 7, pp. 36-43. DOI: 10.5815/ijcnis.2018.07.04 